TITLE:

\title{
Direct versus sequential double ionization of Mg with extreme- ultraviolet radiation
}

\section{AUTHOR(S):}

Nikolopoulos, LAA; Nakajima, T; Lambropoulos, P

\section{CITATION:}

Nikolopoulos, LAA ...[et al]. Direct versus sequential double ionization of Mg with extremeultraviolet radiation. Physical Review Letters 2003, 90(4): 043003.

\section{ISSUE DATE:}

2003-01-31

URL:

http://hdl.handle.net/2433/50425

RIGHT:

Copyright 2003 American Physical Society 


\title{
Direct versus Sequential Double Ionization of Mg with Extreme-Ultraviolet Radiation
}

\author{
L. A. A. Nikolopoulos, ${ }^{1}$ Takashi Nakajima, ${ }^{2}$ and P. Lambropoulos ${ }^{1,3}$ \\ ${ }^{1}$ Institute of Electronic Structure and Laser, F.O.R.TH, P.O. Box 1527, Heraklion 711 10, Crete, Greece \\ ${ }^{2}$ Institute of Advanced Energy, Kyoto University, Gokasho, Uji, Kyoto 611-0011, Japan \\ ${ }^{3}$ Department of Physics, University of Crete, P.O. Box 2208, GR-71110, Crete, Greece
}

(Received 3 May 2002; published 29 January 2003)

\begin{abstract}
We present an ab initio calculation of multiphoton double ionization in atomic magnesium, the first of its type in an atom with a complex core, showing that direct correlated double electron ejection can be studied through analysis of the photoelectron energy spectrum, using currently or upcoming extremeultraviolet radiation sources.
\end{abstract}

DOI: $10.1103 /$ PhysRevLett.90.043003

Recent work on the two-electron (three-body) problem has revealed new layers of physics in the context of fewphoton double ionization [1-7]. In particular, two-photon double ionization of helium has been shown to exhibit features not possible in single-photon double ionization and not easy, if possible at all, to disentangle in strongfield multiphoton ionization under long-wavelength radiation. The picture that appears to be emerging suggests that double ionization, and hence the underlying behavior of the two-electron system, under single-photon excitation on the one hand and multiphoton of high order on the other, represent two extremes with their own features, while the intermediate regime possesses a vast richness of unexplored physics. For example, under two-photon ionization, the judicious choice of wavelength offers the possibility to separate direct from sequential double ionization [2], whose photoelectron energy and angular distribution [5] have shown counterintuitive behavior. Until quite recently, the theoretical analysis of these processes, owing to the lack of sources of appropriate intensity, could have been considered of only academic interest. Ongoing developments on extreme-ultraviolet (XUV) coherent, high intensity and short pulse duration sources, however, place these questions into the mainstream of upcoming new physics.

The two-electron problem beyond $\mathrm{He}, \mathrm{H}^{-}, \mathrm{Ps}^{-}$- the pure Coulombic systems - possesses further dimensions when examined in the context of systems with a complex core, such as the alkaline earths. Here single-photon double ionization for larger photon energies will tend to become overshadowed by Auger processes involving the ejection of electrons below the valence shell, while truly nonperturbative high-order multiphoton double ionization may be virtually unattainable with near-infrared and optical sources such as the Ti:sapphire laser $(780 \mathrm{~nm})$, due to their low ionization potential which would lead to the preponderance of sequential processes. Yet, these very features, that may present obstacles in the single-photon or long-wavelength limit, offer fertile ground for the exploration of this intermediate uncharted regime with the additional opportunity to assess the effects of the core. Given that very little is known about
PACS numbers: $32.80 . \mathrm{Wr}$

double photoionization in those systems, under any circumstances, the maturing of theoretical approaches places such complex tasks within reach, and the availability of appropriate sources, which could even be those based on high-order harmonic generation (HOHG), we have undertaken the study of what could be viewed as a prototype system combining all aspects discussed above.

We consider the $\mathrm{Mg}$ atom under radiation of photon energy $\hbar \omega$ in the range of 10 to $15 \mathrm{eV}$, with specific results to be presented here for $\hbar \omega=14.3 \mathrm{eV}$. One reason for this choice is that this photon energy coincides with the 9th harmonic of the Ti:sapphire laser. At the same time radiation at $\hbar \omega=14.8 \mathrm{eV}$ of intensity up to $10^{14} \mathrm{~W} / \mathrm{cm}^{2}$ and pulse duration 100 fs has already been employed in experiments with the free electron laser source at DESY in Hamburg [8].

Most importantly, however, as shown in Fig. 1, direct two-electron ejection is a two-photon process, while the sequential from the ground state of $\mathrm{Mg}^{+}$is a three-photon process. At the same time, the ion which can be also left in the excited $3 p$ state, will be ionized through the absorption of one more photon, leading thus to a sequential double ionization of second order (two photon). At the end of the pulse, ions resulting from single-photon absorption, but not ionized further, will be left in either the ground $3 s$ or the first excited state $3 p$, the branching ratio depending on intensity and pulse duration. Very distinctive signatures of the behavior of the system are to be found in the photoelectron energy spectra, which can be examined either in themselves or in coincidence with the ions, particularly the doubly ionized one $\left(\mathrm{Mg}^{++}\right)$. Such coincidence measurements, if convenient, although technically demanding, may be helpful in improving the ratio of weak over strong signals. Theoretically, however, all of the important information resides in the photoelectron energy and angular distribution spectra.

To attempt a quantitative assessment of the behavior one needs a reliable basis for the system, including excited ionic states and of course the double continuum. The closed-shell core of $\mathrm{Mg}^{++}\left(2 p^{6}\right)$ is the starting point on which the two-electron states are constructed. Since the photon energy range contemplated for the excitation of 


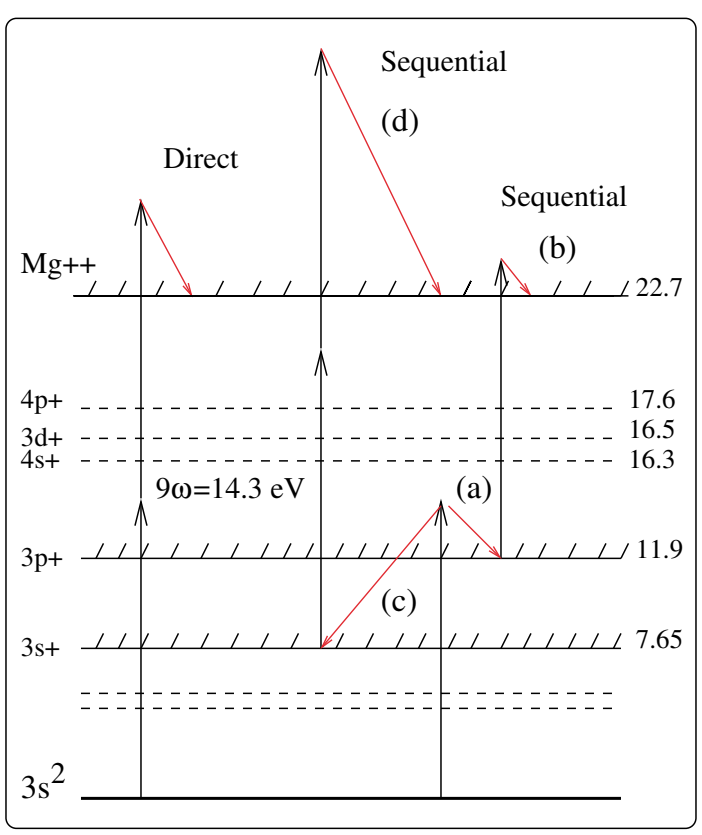

FIG. 1 (color online). Mg energy level diagram (in eV), showing the direct and sequential ionization channels.

the valence electrons is much lower than the lowest excitation energy $(53 \mathrm{eV})$ of the $\mathrm{Mg}^{++}\left(2 p^{6}\right)$ core, we need not be concerned with real excitations of the core. Virtual excitations at such low photon energies but not too high intensity are included in the form of core polarization which is necessary to obtain a satisfactory energy spectrum of Mg. The only information available to us is the structure of the neutral atom, as well as single- and twophoton ionization cross sections leading to lower excited states of $\mathrm{Mg}^{+}$[9-12]. Processes leading to double ejection in magnesium are practically unknown territory. The manner of construction of the basis is not independent of the calculation of the few-photon processes. As already noted above, the inclusion of the core places additional demands on the calculation. To the best of our knowledge, this is the first attempt at a calculation of two-photon double ionization in an atom with a multielectron core. Some related work on single-photon double ionization of beryllium has appeared recently $[13,14]$. Beginning with a Hartree-Fock core $\mathrm{Mg}^{++}\left(2 p^{6}\right)$, we have employed multichannel configuration techniques involving a discretized $L^{2}$ basis consisting of linear combinations of $B$-spline functions. Such a basis proves convenient in performing the summation over intermediate states. The handling and representation of the double continuum, as well as coupled open channels in the manifold of doubly excited autoionizing states, require tools beyond those available in a discretized basis within a radial box of fixed radius with vanishing wave functions at the fixed boundary [1]. As shown recently, a technique based on the idea of a free boundary provides the tools needed for coupled open channels [4]. Having developed and tested these ideas and techniques in the relatively simpler con- text of helium we are now in the position to undertake the problem with a non-Coulombic core.

The Hamiltonian of the magnesium atomic system $H_{a}=\sum_{i=1}^{2}\left[-\frac{1}{2} \nabla_{i}^{2}+V_{\text {eff }}\left(\mathbf{r}_{i}\right)\right]+V\left(\mathbf{r}_{1}, \mathbf{r}_{2}\right)$, where $V_{\text {eff }}\left(\mathbf{r}_{i}\right), i=1,2$ represents the effective potential for the $i$ th electron and the core, and $V\left(\mathbf{r}_{1}, \mathbf{r}_{2}\right)$ is a two-body interaction operator that includes the static Coulomb interaction $1 /\left|\mathbf{r}_{1}-\mathbf{r}_{2}\right|$ as well as a dielectronic effective interaction [9].

At first, we perform a Hartree-Fock calculation for the closed-shell core of magnesium $\left(\mathrm{Mg}^{++}\right)$, deriving thus the effective Hartree-Fock potentials "seen" by an outer electron. At a second stage we solve the Schrödinger equation for the $\mathrm{Mg}^{+}$system. In this case, the effective potential acting on the valence electron is given by $V_{\text {eff }}(r)=V_{l}^{\mathrm{HF}}(r)+\left(\alpha / r^{4}\right)\left\{1-\exp \left[-\left(\frac{r}{r_{l}}\right)^{6}\right]\right\}$, where $\alpha$ is the static polarizability of $\mathrm{Mg}^{++}$and $r_{l}$ is the cutoff radii for the various partial waves $l=0,1,2, \ldots$.

Having produced the $\mathrm{Mg}^{+}$one-electron radial eigenstates $P_{n l}(r)$ for each partial wave $l=0,1,2, \ldots$, with the condition that they vanish at the boundaries of the box, we solve the Schrödinger equation, $H_{a} \Psi^{\Lambda}\left(\mathbf{r}_{1}, \mathbf{r}_{2}\right)=$ $E \Psi^{\Lambda}\left(\mathbf{r}_{1}, \mathbf{r}_{2}\right)$, by expanding the two-electron eigenstates $\Psi^{\Lambda}\left(\mathbf{r}_{1}, \mathbf{r}_{2}, E\right)$ on the basis of the two-electron antisymmetrized orbitals:

$$
\Phi_{n l l^{\prime}}^{\Lambda}\left(\mathbf{r}_{1}, \mathbf{r}_{2}\right)=A_{12} \frac{P_{n l}\left(r_{1}\right)}{r_{1}} \frac{R_{l^{\prime}}\left(r_{2}\right)}{r_{2}} Y_{L M_{L}}\left(\hat{r}_{1}, \hat{r}_{2} ; l, l^{\prime}\right),
$$

where $A_{12}$ is the antisymmetrization operator. We then select the one-electron radial basis function $R_{l}(r)$ to be nonvanishing at the boundaries, thus transforming the Schrödinger equation into a system of algebraic equations for the expansion coefficients $[1,4]$. For this, we use the $B$-spline basis functions [1]. The choice of $R_{l}(r)$ to be nonvanishing at the boundaries provides a controllable density of the continuum spectrum (therefore degeneracy into the continuum is guaranteed), while the two-electron continuum states have been expanded in terms of coretarget states $P_{n l}(r)$ plus a channel function $R_{l}(r)$, as in standard close-coupling scattering theory. This procedure leads to a double continuum spectrum in which the states of one of the electrons are discretized while those of the other are continuous. We produce the initial ground state of magnesium in a box of radius $R=40$ a.u. in which we have included 20 configuration series of the type $n s m s, n p m p, n d m d, n f m f$ with $n$ up to 5 and $m$ up to 60 . The number of $B$-spline basis was 62 and the order 9 . The parameters of the core-polarization potential included were $\alpha=0.491$ for the static polarizability of the core and $1.2,1.335,1.25,1.3$, and 1.1 for the cutoff radii for the $l=0,1,2,3,4$ partial waves, respectively. This gives for the singly ionized magnesium $E_{3 s}=-14.999 \mathrm{eV}$ and for the neutral magnesium ground state equal to $E_{3 s^{2}}=-22.65 \mathrm{eV}$. In terms of the ionization potential of neutral magnesium this corresponds to $7.6499 \mathrm{eV}$ as compared to the experimental value $7.6462 \mathrm{eV}$ [9]. 
The two-photon direct double ionization cross section of $\mathrm{Mg}$ is shown in Fig. 2 for the range of photon energies of interest in this paper. Given the demand of the calculations, and the relative agreement between length and velocity gauge, we have obtained, we believe that those cross sections are within the order of magnitude of the real values. Compared to an earlier analogous result for $\mathrm{He}$, these cross sections differ in magnitude. This must be the result of the different dipole matrix elements expected in $\mathrm{Mg}$ as well as the lower ionization potential. The two are of course related to each other and to the larger size of the atom. The significance of this cross section to the observability of the processes is discussed below.

The time-dependent Hamiltonian of the system is $H=$ $H_{a}+D(t)$ where the operator $D(t)$ is the interaction between the atom and the external laser field in the dipole approximation. Following a standard procedure, we expand the time-dependent wave function of the atomic system in the basis of the field-free two-electron states, including the ground state of magnesium $|g\rangle$, the singly ionized continuum states $\left|c_{i} ; \varepsilon_{i}\right\rangle,\left[c_{2} \rightarrow \mathrm{Mg}^{+}(3 s), c_{3} \rightarrow\right.$ $\left.\operatorname{Mg}^{+}(3 p)\right]$, and the doubly ionized states $\left|f_{i} ; \varepsilon_{i}, \varepsilon_{i}^{\prime}\right\rangle\left(f_{1} \rightarrow\right.$ $\mathrm{Mg}^{++}$from the two-photon direct absorption from the ground state, while $f_{2},\left(f_{3}\right) \rightarrow \mathrm{Mg}^{++}$from the two(one-) photon absorption from the excited state $\left.\mathrm{Mg}^{+}(3 s)\left[\mathrm{Mg}^{+}(3 p)\right]\right)$. By inserting the state function $\psi(t)$ into the time-dependent Schrödinger equation, $\partial|\psi(t)\rangle / \partial t=-i\left[H_{0}+D(t)\right]|\psi(t)\rangle$, we obtain a system of first-order differential equations for the timedependent expansion coefficients, namely,

$$
\begin{gathered}
\dot{u}_{g}=-\frac{1}{2}\left[\gamma_{g, f_{1}}+\gamma_{g, c_{2}}+\gamma_{g, c_{3}}\right] u_{g}, \\
\dot{u}_{c_{i}}=-i\left[E_{c_{i}}-i \frac{\gamma_{c_{i}, f_{i}}}{2}\right] u_{c_{i}}-i D_{c_{i} g} u_{g} \quad(i=2,3), \\
\dot{u}_{f_{i}}=-i E_{f_{i}} u_{f_{i}}-i D_{f_{i j}} u_{j}, \quad(i=1,2,3),
\end{gathered}
$$

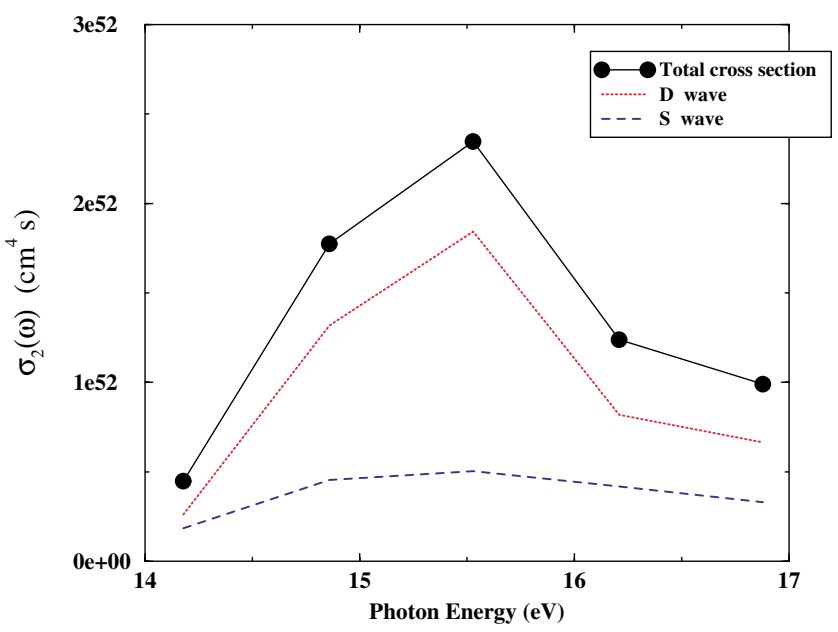

FIG. 2 (color online). Direct two-photon double ionization cross section in the range $14-17 \mathrm{eV}$. with $j=\mathrm{g}$ for $i=1$ while $j=c_{i}$ for $i=2,3$ and with $E_{f_{1}}=\epsilon_{1}+\epsilon_{1}^{\prime}-\left(E_{3 s^{2}}+2 \hbar \omega\right), E_{c_{2}}=E_{3 s}+\epsilon_{2}-$ $\left(E_{3 s^{2}}+\hbar \omega\right), E_{c_{3}}=E_{3 p}+\epsilon_{3}-\left(E_{3 s^{2}}+\hbar \omega\right), E_{f_{2}}=\epsilon_{2}+$ $\epsilon_{2}^{\prime}-\left(E_{3 s^{2}}+3 \hbar \omega\right)$, and $E_{f_{3}}=\epsilon_{3}+\epsilon_{3}^{\prime}-\left(E_{3 s^{2}}+2 \hbar \omega\right)$. The ionization rates into various continua are defined in terms of the corresponding dipole matrix elements as $\gamma_{g, f_{1}}=2 \pi \int d \varepsilon_{1}\left|D_{f_{1} g}\left(\varepsilon_{1}\right)\right|^{2}, \gamma_{c_{i}, f_{i}}=2 \pi\left|D_{f_{i} c_{i}}\right|^{2}, i=2,3$, and $\gamma_{g, c_{i}}=2 \pi\left|D_{c_{i} g}\right|^{2}$. Propagating the system of equations in time, with a realistic temporal pulse shape, we calculate the photoelectron spectrum, at the end of the pulse, associated with the double continuum as

$$
\frac{d P_{f_{i}}}{d \epsilon_{i}}=\int d \epsilon_{i}^{\prime}\left|u_{f_{i}}\left(\epsilon_{i}, \epsilon_{i}^{\prime}, t \rightarrow \infty\right)\right|^{2} \quad(i=1,2,3)
$$

and the photoelectron spectrum associated with the single continuum as $d P_{c_{i}} / d \epsilon_{i}=\left|u_{c_{i}}\left(\epsilon_{i}, t \rightarrow \infty\right)\right|^{2}(i=$ $2,3)$. The photoelectron energy spectrum is shown in Fig. 3. The most interesting feature of this spectrum is the separation of parts of the spectrum of photoelectrons resulting from direct double ejection from the peaks resulting from single-photon ionization of $\mathrm{Mg}$ leaving the ion either in the ground $3 s$ state or the first excited state $3 p$, as well as the two additional peaks corresponding to two-photon ionization of $\mathrm{Mg}^{+}(3 s) \rightarrow \mathrm{Mg}^{++}$and single-photon ionization $\mathrm{Mg}^{+}(3 p) \rightarrow \mathrm{Mg}^{++}$. The energy spectrum of direct double ionization is practically flat, which is in agreement with an earlier result on helium [2]. It can be argued that true direct double ionization can be found only in two- or more-photon ionization, since in single-photon double ionization the photon must "first" be absorbed by one of the electrons which "then" interacts with the other, which in some sense represents a sequential process. Although different from the sequential in multiphoton ionization, where the second electron is ejected by photoabsorption from the ground state of the ion, still it is quite different from the direct in two-photon ionization which can take place even if the electrons were noninteracting particles. A further new feature here is the possibility of two electrons in the continuum with the same angular momentum, namely, $p$ in this case. The spectra in Fig. 3 have been calculated for a pulse of duration and intensity currently available, with some effort, through sources of HOHG. Although the two high yield peaks (corresponding to single-photon ionization of the neutral) provide signals larger than the direct by a few orders of magnitude, they can in principle be discriminated against the direct, depending on the limits of saturation of the detectors, the discussion of which is beyond the scope of this paper. The main point of the results summarized in Fig. 3 is the first, to the best of our knowledge, documentation of direct and sequential double ionization in the context of a complex atom, involving at the same time excitation of the residual core $\mathrm{Mg}^{+}$, and all of this in a realistic and quantitative scenario, within reach of current XUV sources of short duration and sufficient intensity. In the same figure we show the photoelectron signals into each of the channels 


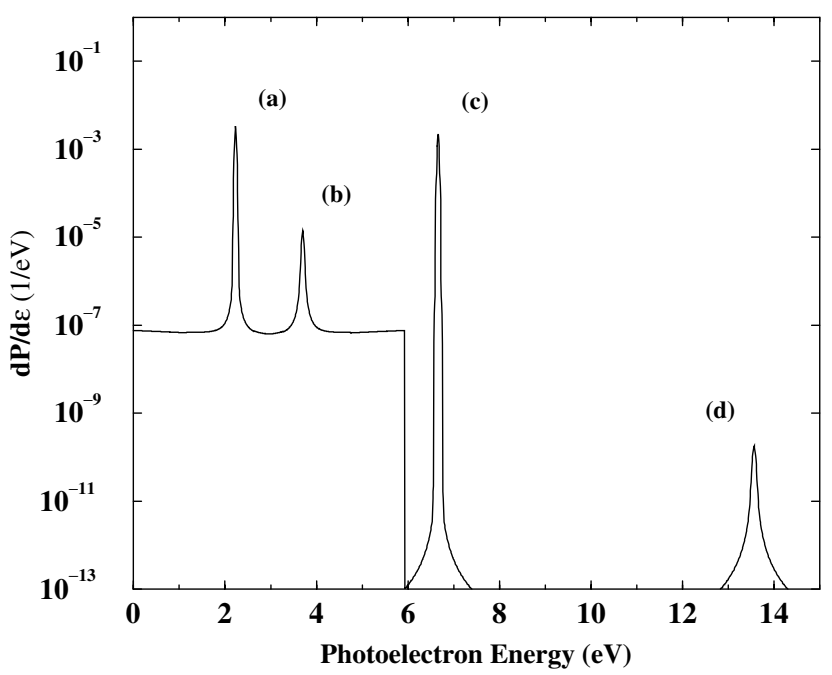

FIG. 3. Photoelectron energy spectrum for the 9th harmonic of the Ti:sapphire laser $(780 \mathrm{~nm})$ with a $50 \mathrm{fs}$, Gaussian pulse and peak. Intensity $10^{12} \mathrm{~W} / \mathrm{cm}^{2}$. The flat part of the spectrum between 0 and $5.9 \mathrm{eV}$ represents the probability for the direct double ejection as a function of the kinetic energy of the one of the photoelectron, at the end of the pulse, while the peaks correspond to sequential processes indicated in Fig. 1.

indicated in Fig. 1. We have taken a Gaussian temporal pulse shape of duration $50 \mathrm{fs}$ at FWHM, peak intensity $10^{12} \mathrm{~W} / \mathrm{cm}^{2}$ and photon energy $14.3 \mathrm{eV}$. The flat part of the curve extending from 0 to $5.9 \mathrm{eV}$ represents direct double ionization. We have peaks at 6.66 and $13.57 \mathrm{eV}$ for the sequential $(1+2)$-photon double ionization via $\mathrm{Mg}^{+}(3 s)$ [peaks $(c)$ and $(d)$, respectively] and 2.27 and $3.7 \mathrm{eV}$ for the sequential $(1+1)$-photon double ionization via $\mathrm{Mg}^{+}(3 p)$ [peaks $(a)$ and $(b)$ ]. The photoelectrons originating from the singly ionized $\mathrm{Mg}^{+}(3 s)$ and $\mathrm{Mg}^{+}(3 p)$ cores are also counted as a $\mathrm{Mg}^{++}$signal. Since the sequential double ionization via $\mathrm{Mg}^{+}(3 p)$ is a $(1+1)$-photon process, our calculation has shown that about $10^{-3}$ of $\mathrm{Mg}^{+}(3 p)$ absorbs an additional photon to eject the second electron [peak $(b)$ ]. The sequential double ionization via $\mathrm{Mg}^{+}(3 s)$ is a $(1+2)$-photon process, and much less $\mathrm{Mg}^{+}(3 s)$ is expected to eject the second electron, as demonstrated by the magnitude of the peak at $13.57 \mathrm{eV}$ [peak (d)]. We note, in passing, that the absorption of one photon leaves the ion in the $\mathrm{Mg}^{+}(3 s)$ and $\mathrm{Mg}^{+}(3 p)$ with roughly equal probabilities, which made it necessary in account for the branching ratio to $\mathrm{Mg}^{+}(3 p)$. Incidentally, the widths of the sequential peaks are determined by the ionization width of the respective ionic state and the laser bandwidth, the latter giving the dominant contribution.

From the result shown in Fig. 3 it is clear that the photoelectron signals with energies of 0 to $2 \mathrm{eV}$ and 4 to $5.9 \mathrm{eV}$ are practically due to the direct double ionization. And this is the unique feature of the combination of atomic system with wavelength. A number of subtle features are reflected in the photoelectron angular distribu- tion, in analogy to those found earlier in helium [5], but space does not allow their discussion here. It will suffice to mention that, as already alluded to, this process offers the possibility to study photoelectrons in the double continuum having the same orbital angular momentum $(p)$ and nearly equal or very unequal kinetic energies, thus providing a glimpse into otherwise difficult to access aspect of correlation in the continuum.

Finally, a word of caution on the relation between timedependent calculations and cross sections: Two-photon ionization cross sections exhibit deep minima (theoretically zero) between intermediate states. The temporal shape, the total duration, and, in particular, the rise time for pulses other than smooth, e.g., trapezoidal versus Gaussian, can critically affect the ionization yield by orders of magnitude, leading thus to widely varying values of the cross sections extracted from the ionization yield. This appears to be the reason for differences between calculations based on different methods noted in a recent paper dealing with the analogous problem in helium [7]. Cognizant of that sensitivity, we have here employed Gaussian shapes which avoid such artifacts, in addition to being realistic models of experimental pulses.

The work by T. N. was supported by a Grant-in-Aid for scientific research from the Ministry of Education and Science of Japan. T. N. also acknowledges the hospitality from IESL-FORTH during his stay where part of this work was carried out.

[1] P. Lambropoulos, P. Maragakis, and Jian Zhang, Phys. Rep. 305, 203 (1998).

[2] M. A. Kornberg and P. Lambropoulos, J. Phys. B 32, L603 (1999).

[3] D. Dundas et al., J. Phys. B 32, L231 (1999); J. S. Parker et al., J. Phys. B 33, L691 (2000); J. S. Parker et al., J. Phys. B 34, L69 (2001).

[4] L. A. A. Nikolopoulos and P. Lambropoulos, J. Phys. B 34, 545 (2001).

[5] M. G. Makris, L. A. A. Nikolopoulos, and P. Lambropoulos, Europhys. Lett. 54, 722 (2001).

[6] T. Mercouris, C. Haritos, and C. A. Nikolaides, J. Phys. B 34, 3789-3811 (2001).

[7] J. Colgan and M. S. Pindzola, Phys. Rev. Lett. 88, 173002 (2002).

[8] V. Ayvazyan et al., Phys. Rev. Lett. 88, 104802 (2002); H. Wabnitz et al., Nature (London) 420, 482 (2002).

[9] S. Mengali and R. Moccia, J. Phys. B 29, 1613 (1996).

[10] H. S. Fung et al., Phys. Rev. A 64, 052716 (2001).

[11] A. Reber et al., Phys. Rev. A 65, 063413 (2002).

[12] E. Luc-Koening et al., J. Phys. B 30, 5213 (1997).

[13] J. Colgan and M.S. Pindzola, Phys. Rev. A 65, 022709 (2002).

[14] A. S. Kheifets and Igor Bray, Phys. Rev. A 65, 012710 (2002). 